\title{
MAGNETIC FIELD INFLUENCE ON AUGER EFFECT ON SHALLOW DONORS IN $\mathrm{CdF}_{2}: \mathrm{Mn}^{2+} \mathbb{L U M I N E S C E N C E}$
}

\author{
A. Kamińska and A. Suchocki \\ Institute of Physics, Polish Academy of Sciences \\ Al. Lotników 32/46, 02-668 Warsaw, Poland
}

\begin{abstract}
Direct observation of the suppression of the Auger effect on shallow donors by the magnetic field in the luminescence of manganese ions in semiconducting $\mathrm{CdF}_{2}: \mathrm{Mn}$ crystals is presented. The magnetic field decreases the probability of the Auger effect, which is spin-dependent energy transfer from the manganese ions to the electrons occupying shallow donors. This results in the increase in the decay times of the luminescence.
\end{abstract}

PACS numbers: 73.20.Hb, 73.50.Gr

The mechanism of the nonradiative recombination of the excited states of impurities in insulating crystals is a topic of interest in solid-state physics. One of them is energy migration on the system of excited ions and energy transfer to different types of defects.

In semiconductors, the presence of carriers opens a new recombination channel - the Auger effect (AE). The excitation energy of the localized dopant can be nonradiatively transferred either to the free carrier or the carrier bound to some defect center. In any case the energy accepting carrier is promoted during the $\mathrm{AE}$ high into the respective band (the conduction band for the electrons and valence band for the holes). This effect has been found in $\mathrm{CdF}_{2}$ crystals doped with $\mathrm{Mn}^{2+}$ and $\mathrm{Y}^{3+}$ ions [1]. The Coulomb interaction, responsible for the $\mathrm{AE}$, conserves the spin and its projection. Since magnetic impurity is involved in this $\mathrm{AE}$, its probability should be dependent on spin polarization of particles participating in the process.

$\mathrm{Mn}^{2+}\left(3 d^{5}\right)$ in $\mathrm{CdF}_{2}$ is a very efficient activator of a blue-green luminescence $(2.44 \mathrm{eV})$ corresponding to transitions from the first excited state ${ }^{4} T_{1 \mathrm{~g}}\left({ }^{4} G\right)$ to the ${ }^{6} A_{1 \mathrm{~g}}\left({ }^{6} S\right)$ ground state of the $\mathrm{Mn}^{2+}$ ion in a centrosymmetric environment. The radiative decay time $\tau_{\mathrm{r}}$ of the $\mathrm{Mn}^{2+}$ luminescence in $\mathrm{CdF}_{2}$ is equal to $158 \mathrm{~ms}$ at $4.2 \mathrm{~K}$ [1].

$\mathrm{CdF}_{2}$ is one of the crystals with the fluorite structure. In opposite to the other fluorite crystals, which are good insulators, $\mathrm{CdF}_{2}$ may be converted to a low-resistance $n$-type semiconductor by doping with transition metal or rare earth 
ions (substituting $\mathrm{Cd}$ ion). The crystal used in our experiment was doped with $1 \mathrm{~mol} \%$ of $\mathrm{Mn}$ and $0.05 \mathrm{~mol} \%$ of $\mathrm{Y}$. It was converted to the conductivity state by the annealing in $\mathrm{H}_{2}$ atmosphere (to remove the interstitial compensating fluorine ions). Free electrons liberated at the surface were trapped by $\mathrm{Y}$ ions and neutral shallow donors were formed. Their activation energy is about $110 \mathrm{meV}$ for low concentration of donors [2].

The sample was placed into the superconducting Oxford magnet system, in the range of magnetic field up to 7 teslas. The luminescence was excited by the 337.1-nm line of a pulsed nitrogen laser (excitation pulse duration of about $10 \mathrm{~ns}$ ), which is resonant with the intrashell $3 d^{5}$ transitions of $\mathrm{Mn}^{2+}$ ions. The decay kinetics were measured with the use of Multichannel Scaller/Averager SR 430. The measurements were performed at $2.2 \mathrm{~K}$.

In the presence of shallow donors the $\mathrm{Mn}^{2+}$ luminescence becomes weaker and the decay time shorter because of $\mathrm{AE}$ - the excitation energy transfer from the $\mathrm{Mn}^{2+}$ ions to the electrons. At temperatures above $40 \mathrm{~K}$ the free electron $\mathrm{AE}$ dominates. Its probability is proportional to the free carriers concentration and this effect is governed by the dipole-dipole interaction. Below $40 \mathrm{~K}$, when practically all electrons are frozen out at the shallow donors, the energy is transferred to the donors, this causes their ionization with the electrons excited high into the conduction band. It is a resonant energy transfer, since the luminescence spectrum of the sensitizer ion $\left(\mathrm{Mn}^{2+}\right)$ overlaps the absorption spectrum of the activator ions (in our case - the photoionization spectrum of the shallow donors). Because of continuous character of the photoionization spectrum of the shallow $\mathrm{Y}$ donors this resonance condition is fulfilled, although the photoionization energy of shallow donor is much lower than the energy of the $\mathrm{Mn}^{2+}$ luminescence.

It has been found that the $\mathrm{AE}$ due to the electron bound on shallow donors in $\mathrm{CdF}_{2}:(\mathrm{Mn}, \mathrm{Y})$ is dominated by the exchange interaction [1]. Therefore the energy transfer probability $P$ is exponentially dependent on the distance $R$, between the interacting centers

$$
P=P_{0} \exp \left(-\frac{2 R}{a_{\mathrm{B}}}\right)=\frac{1}{\tau_{\mathrm{r}}} \mathrm{e}^{\gamma} \exp \left(-\frac{2 R}{a_{\mathrm{B}}}\right),
$$

where $\tau_{r}$ is the radiative lifetime of sensitizer ions, $a_{B}$ is the shallow donor Bohr radius $\left(a_{\mathrm{B}}=7 \AA\right.$ for $\mathrm{CdF}_{2}$ [2]). The $\gamma$ parameter describes the strength of the exchange interaction and it is equal to $\gamma=2 R_{0} / a_{\mathrm{B}}$, where $R_{0}$ is the critical distance, at which the $\mathrm{AE}$ rate equals the radiative probability.

The value of $\gamma$ depends on the magnetic field and in its absence is equal to 14 [1]. This value can be found by the fit of Inokuti-Hirayama model [3], which describes the luminescence decay kinetics. Figure 1 shows the dependence of $\mathrm{AE}$ probability on the magnetic field, derived from the fit of the Inokuti-Hirayama model to the experimental luminescence decay kinetics.

In the external magnetic field the energy levels of $\mathrm{Mn}^{2+}$ ions split into Zeeman sublevels. The probability of Auger effect depends on the spin polarization of shallow donors and manganese ions. In the magnetic field all thermalized electrons have the same spin-down polarization at low temperatures. The Coulomb interaction responsible for the Auger effect conserves the total spin and its projection. Therefore only transitions between the Zeeman sublevels of the ${ }^{4} T_{1 \mathrm{~g}}$ and 


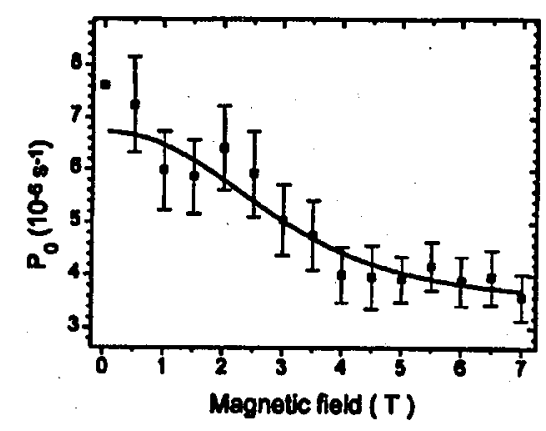

Fig. 1. The magnetic field dependence of the Auger effect probability on shallow donors in the $\mathrm{CdF}_{2}: \mathrm{Mn}$ luminescence. The solid line is the theoretical fit to the data.

${ }^{6} A_{1 \mathrm{~g}}$ states of $\mathrm{Mn}^{2+}$ ion with the same spin projection are allowed. The transitions between the sublevels with different spin projection are forbidden in the strong magnetic field since that would require different polarization of the spin of electron on the shallow donor and in the conduction band. This is the reason for the suppression of the $\mathrm{AE}$ in the presence of magnetic field.

The dependence of the probability of the Auger effect on the spin polarization of the particles can be found in the general form if we neglect any spin-orbital coupling in the system. According to the theoretical model of Nawrocki et al. [4] it is given by

$$
P_{\mathrm{A}}(B) \propto \sum_{\sigma, \sigma^{\prime}= \pm 1 / 2} P_{\mathrm{e} \sigma}(B) P_{\mathrm{e} \sigma^{\prime}}(B) \sum_{\mu=3 / 2}^{3 / 2} C_{\sigma, \sigma^{\prime}}^{\mu} p_{\mu}(B)
$$

where $P_{\mathrm{e} \sigma}(B)$ is the probability for the electron to be in the state with spin projection $\sigma$

$$
P_{\mathrm{e} \sigma}(B)=Q_{\mathrm{e}}^{-1} \exp \left(-\frac{g \mu_{\mathrm{B}} B}{k T} \sigma\right), \quad Q_{\mathrm{e}}=\sum_{\sigma= \pm 1 / 2} \exp \left(-\frac{g \mu_{\mathrm{B}} B}{k T} \sigma\right) .
$$

The relative probabilities of the Auger transition for different initial spin polarizations $C^{\mu} \sigma \sigma^{\prime}$ can be calculated from the Clebsh-Gordan coefficients.

The probability for the $\mathrm{Mn}^{2+}$ ion to be in the state with spin projection $\mu, p_{\mu}(B)$ is expressed by

$$
\begin{aligned}
& p_{\mu}(B)=\frac{\exp (-\lambda \mu)}{Q_{\mathrm{Mn}}}, \quad \lambda=\frac{g_{\mathrm{Mn}} \mu_{\mathrm{B}} B}{k T}, \\
& Q_{\mathrm{Mn}}=\sum_{\mu=-3 / 2}^{3 / 2} \exp (-\lambda \mu)=\frac{\sinh (2 \lambda)}{\sinh (\lambda / 2)} .
\end{aligned}
$$

The solid line in Fig. 1 represents the computer fit of Eq. (2) to the experimental data of the AE probability dependence on the external magnetic field $B$.

In conclusion, we have observed the suppression of the $\mathrm{AE}$ by the magnetic field applied to the $\mathrm{CdF}_{2}:(\mathrm{Mn}, \mathrm{Y})$ crystal, which is explained by spin polarization 
of electrons involved in the $\mathrm{AE}$ and $\mathrm{Mn}^{2+}$ ions. The presented theory is in very. good agreement with the experimental data.

The authors are indebted to Dr. Marek Potemski of IMP-CNRS High Magnetic Field Laboratory in Grenoble, France, for valuable discussion.

\section{References}

[1] A. Suchocki, J.M. Langer, Phys. Rev. B 39, 7905 (1989).

[2] J.M. Langer, T. Langer, G.L. Pearson, B. Krukowska-Fulde, U. Piekara, Phys. Status Solidi $B$ 66, 537 (1974).

[3] M. Inokuti, F. Hirayama, J. Chem. Phys. 43, 1978 (1965).

[4] M. Nawrocki, Yu.G. Rubo, J.P. Lascaray, D. Coquillat, Phys. Rev. B 52, R2241 (1995). 